\title{
De la recherche scientifique et de la foi chrétienne
}

\author{
Philippe Deterre
}

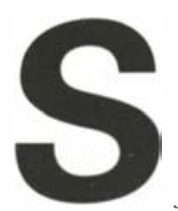

cience et religion forment depuis Galilée un nœud de querelles persistantes et constituent jusqu'à aujourd'hui un vieux classique de la presse et de l'édition, y compris scientifique [1]. Sur ce front, l'astrophysique est très sollicitée [2-4], mais aussi la biologie, notamment depuis Darwin. Une question souvent posée est de savoir si les postulats et les résultats de la science laissent ou non une place à Dieu et aux religions. D'un côté, si la physique peut comprendre l'univers et son commencement, si l'évolution biologique peut expliquer la diversité du vivant, si les neurosciences peuvent rendre compte de ce qu'est l'esprit humain, alors Dieu devient inutile et sans intérêt. D'un autre côté, certains, s'appuyant sur les paradoxes classiques de la mécanique quantique, redonnent une chance à Dieu ou à certaines religions $[5,6]$. Dans tous les cas, il s'agit d'opposer, ou de concilier, les représentations du monde données par la Science avec celles qui sont livrées par les Ecritures. Mon propos ici est une tentative pour déplacer le problème. Savoir si les sciences contemporaines laissent, ou ne laissent pas, une place à Dieu ne me semble pas être une question essentielle. Et ce pour - au moins trois raisons: d'abord, parce que, par méthode, les sciences se situent à l'extérieur de la question religieuse; ensuite, parce que la théologie contemporaine ne lit plus les récits de création comme une source d'information sur le début du monde; et, enfin, parce qu'un Dieu ou une religion qui n'auraient pour seules raisons que les lacunes provisoires, et sans cesse déplacées, de l'explication scientifique seraient bien peu dignes de foi et d'intérêt. Prêtre catholique et chercheur scientifique*, ma réflexion se situe sur une ligne différente et plus modeste. Ma proposition est de mettre la foi chrétienne en regard, non pas des résultats de la Science et de ses théories, mais de la pratique de ceux qui "font" la Science, à savoir les chercheurs. La question est alors celle de l'éventuelle pertinence de la tradition croyante et chrétienne pour le chercheur scientifique. Ce texte n'engage que son auteur, qui remercie le comité de rédaction de médecine/sciences de la tribune qui lui est proposée.

\section{Le sujet de la recherche}

Mon point de départ est une proposition banale: il n'y a pas de recherche scientifique sans chercheurs. Les résultats de la Science peuvent bien être présentés de manière impersonnelle - "sans sujet $»$, la recherche n'en reste

\footnotetext{
* Ingénievar de fonnation, j'ai d'abord tratuaillé en physique du solide azuant une reconversion en biologie. J'ai passé ma thèse dlitat en 1983, juste un mois aucant d'être ordonné prêtre. Je suis membre de ce groupe de prêtres qui ont un tratuail professionnel et qu'on appelle "Mission de France".
}

pas moins une aventure humaine qui dépend historiquement de l'intuition d'un tel, du travail de tel autre, du pari fait à tel moment, de la constitution de telle équipe, de l'existence de tel institut, etc. Cha-' cune des récentes avancées de la biologie moderne pourrait ici servir d'exemple à cette assertion. Certes, la sociologie des sciences nous montre combien la recherche scientifique peut être le théâtre de jeux de prestige, de persuasion et de modes, qui jouent un grand rôle dans l'adoption de tel ou tel résultat et dans le financement de tel ou tel genre de recherche [7]. Certes, l'analyse linguistique a bien décrit la structure de toute publication scientifique comme une stratégie pour "faire savoir" en vue de "faire croire" $[8,9]$. Mais, finalement, tout chercheur honnête sait bien tout cela, de l'intérieur. Il n'en reste pas moins que la véritable recherche scientifique n'est pas seulement déterminée par le pouvoir du prestige, par celui de l'économique ou par la pression des sujets à la mode. L'exigence de vérité qui est portée dans les laboratoires de recherche dignes de ce nom n'est pas soumise au seul principe de rendement et de la publication à tout prix. Plus largement, il faut bien constater qu'il y a un indéterminé, un imprévisible dans la recherche, et c'est bien cela, me semble-t-il, qui en fait le sel et l'intérêt pour ceux dont c'est la profession de chercher. 
I.e travail du chercheur n'est pas seulement le développement d'un programme préétabli. Il consiste, au sein d'une problématique déterminée et bien choisie, à poser les bonnes questions et, surtout, à savoir s'interroger sur les faits qui apparaissent insolites. Il y a quelque chose du guetteur dans le chercheur: guetter l'idée neuve et féconde, le résultat qui ne "cadre pas" et peut faire basculer tout le travail en cours. Que l'on pense ici à la découverte de la pénicilline, ou à celle des gènes mosaïques. Les avancées majeures sont souvent des heureuses surprises issues d'une observation fortuite sur laquelle une équipe a su s'interroger et travailler. Il n'y a pas de recherche efficace sans travail, mais le travail ne suffit pas: voilà bien ce qui rend ce métier si difficile et si passionnant. Il n'y a pas de chercheur isolé : tout chercheur avisé le sait bien. Il n'y a de vérité en science que dans la vérification. Vérification de l'expérience par l'expérience, vérification du travail des uns par les autres, par la reconnaissance des uns par les autres. Cela s'opère dès le laboratoire, où l'expérimentateur rend compte à ses collègues des résultats qu'il a obtenus et de l'interprétation qu'il en donne, jusqu'au congrès scientifique et la publication d'un article, après avis donné par les "pairs". La recherche scientifique est le lieu d'action de sujets en travail et en constante communication au sein de ce qu'on appelle à juste titre la "communauté scientifique".

\section{La "foi" du chercheur}

Cette description de la recherche comme une entreprise à la fois individuelle et collective de vigilance et de critique pour faire émerger de nouveaux modèles - de nouveaux paradigmes, dit-on parfois - ne suffit pourtant pas à rendre compte de la passion des chercheurs. Dans ses mémoires, François Jacob situe bien le niveau de l'interrogation:

"Par quelle nécessité des hommes mettent-ils tant de passion, prennent-ils tant de plaisir à vouloir $\mathrm{m} / \mathrm{s} n^{\circ}$ 6-7 vol. 10, juin-juillet 94 éternellement explorer le monde, l'interroger? A cette question, ceux qui aiment la Science répondent: par curiosité, par désir de s'approprier la nature, d'améliorer le sort de l'homme. Ceux qui n'aiment pas la Science disent : par ambition, par volonté de puissance, amour de la gloire ou même cupidité. Mais ce n'est pas tout. Il y a des facteurs plus profonds. Il y a la tentative, la tentation de comprendre un monde qui se dérobe. La révolte contre la solitude. Contre une réalité qui vous échappe, qui vous ignore et sans laquelle il n'y a pas de vie. Une exigence métaphysique de cohérence et d'unité, dans un univers qu'on cherche à posséder mais qu'on ne parvient pas même à saisir [10]. " Il y a effectivement du métaphysique dans la motivation du chercheur, en tout cas en ce sens précis de la recherche de cohérence et d'unité dans le monde. ('est cette recherche inextinguible qui appelle l'explication claire, qui guide dans les méandres des raisonnements et des techniques nécessaires. ('est cette force qui relance en permanence et juge toute l'aventure et son jeu. I a méthode scientifique n'est qu'une médiation entre le monde qui se présente et ce désir humain de connaissance, cette révolte contre l'incohérence. La méthode n'avance pas seule : elle dépend de cette pulsion fondamentale, qu'avec les précautions nécessaires j'appelle une "foi ", en ce sens qu'une certaine confiance dans l'opération scientifique est nécessaire pour avancer, pour continuer de chercher cette cohérence et cette unité. Une certaine confiance primordiale dans l'intelligibilité du monde et dans le travail du sujet humain me semble à l'œuvre dans le quotidien de la recherche scientifique, qui permet de croire que, derrière l'apparent chaos du monde et sa diversité, le travail patient d'analyse, de critique et de veille livrera quelques clés d'explication supplémentaires. Et cela suppose une exigence, une rigueur et une humble détermination - une éthique dirait-on aujourd'hui* [11] — qui me semble être le plus précieux de ce que j'ai rencontré dans les diffërents laboratoires où j'ai eu la chance de travailler.

Cette mise en perspective de ce qui est à l'oeuvre dans la recherche scientifique n'est probablement pas originale. Elle est sans doute discutable. Elle repose en tout cas sur une conception du sujet humain de "l'être au monde" comme disent les philosophes - qui est compatible avec l'existence d'un sujet croyant. L'homme ainsi pensé - scientifique et/ou croyant - est un sujet qui ne cesse de se remettre au travail, travail de déchiffrement du réel, travail de lecture et d'écriture, travail de recherche pour guetter le neuf et savoir saisir "ce qui arrive ", travail de vérification permanente, à la fois constructif et critique. Et ce travail suppose une certaine confiance, une certaine "foi ", en ce que le chemin est possible et que la tâche n'est pas vaine.

\section{L'interrogation chrétienne}

Cette explicitation étant faite, il est possible d'indiquer quelques-uns des points de contact possibles entre la recherche scientifique et la foi chrétienne. Je le ferai en partant des notions de création, d'incarnation et de sabbat.

On pourra interpréter la tâche humaine de déchiffrement du réel comme une concurrence faite à Dieu (c'est d'ailleurs le sens qu'en donne François Jacob dans la suite du texte cité ci-dessus). L'interprétation chrétienne, comme l'interprétation juive, est différente: selon elle, la quête de connaissance n'est pas une usurpation d'un quelconque pouvoir divin, mais bien d'abord une participation à la geste divine de création qui, selon le premier chapitre du livre de la Genèse, est de "mettre en ordre" le chaos cosmique par séparations et distinctions

\footnotetext{
* Il faut iri remal re justice a Jacques Momod d'aroir été le premier ì parler d'éthique - même s'il s'agissait d'une très rigoriste "éthique de la connaissance" - i un moment one cela était bien loin d'être "ci la mode".
} 
successives*. On retrouve là un thème classique en théologie: par le langage qui lui permet de nommer ce qui existe et ce qui arrive, par le travail qui lui permet de modifier et de maîtriser, l'homme participe à la création. La théologie chrétienne valorise ainsi le travail de différenciation et de mise en rapport que le scientifique opère pour ordonner et comprendre l'univers. En mettant à jour les lois d'agencement des éléments du monde, en déterminant les niveaux pertinents d'analyse et d'intervention, l'homme est cocréateur. Il a été d'ailleurs souvent souligné combien la tradition judéochrétienne, qui sépare le Créateur et le monde créé, a été historiquement féconde au moment de l'émergence des sciences modernes. En effet, cette séparation créateur/créature "désacralise" le monde qui se trouve ainsi objet possible d'une investigation qui n'offense pas Dieu. Sous l'influence du rationalisme grec, cette investigation a pu devenir science expérimentale $[13,14]$. Selon ce point de vue, la foi du croyant est donc tout sauf un obstacle au travail scientifique: elle en surdétermine la motivation et la nécessité. Connaître ce qui est pour en rendre compte et grâces devant Celui qui est.

Après la création**, on peut trouver dans ce que la tradition chrétienne appelle l'incarnation un autre point de convergence possible avec la pratique scientifique. Tout chercheur sait bien aujourd'hui qu'il n'y a de travail fécond en science qu'en investissant son énergie dans un domaine particulier, qui apparaît très exigu à celui qui le découvre pour la première fois. Il n'y a pas d'autre voie pour une science opératoire que celle d'accepter de travailler selon un angle précis sur un aspect particulier d'un problème

\footnotetext{
* Cela constitue d'ailleurs un des thèmes les plus importants de l'ensemble du Prennier Testament, mis en lumière, entre autres, par l'exégète Paul Beauchamp in [12].

** On aura remarqué qu'il n'a pos élé question ici de la création comme commencement de l'univers, et donc d'une éventuelle corrélation avec la
}

bien défini, d'accepter d'y engager là tout son jeu, tout son travail d'imagination, de critique, de doute et d'expérience. Il n'y a de science qu'appuyée sur ce point particulier. C'est à partir de là seulement que le travail d'un scientifique peut avoir une valeur plus générale. Dans une certaine mesure, on peut dire la même chose du chrétien : à cause du Jésus historique de Palestine, il n'y a désormais pas d'autre lieu pour la rencontre avec le Dieu transcendant que le chemin particulier de l'homme. Parce que Dieu a pris corps en une culture et une histoire particulières, il n'y a plus de condition humaine qui soit trop petite ou trop singulière. C'est là, et non pas dans un quelconque état idéal paradisiaque, état détaché de toute passion ou état second hors du corps, que le bonheur - la Béatitude authentique - est possible. Le scientifique d'aujourd'hui est dans un paysage éclaté : le temps des encyclopédistes est terminé, il n'y a plus guère de synthèse possible entre les diverses sciences particulières. Pour le chrétien, cette "finitude" n'est pas un pis-aller, elle est à vivre avec bonheur et sans nostalgie: c'est là, dans son travail de création scientifique, de maîtrise responsable et de compréhension de la réalité, qu’il peut rejoindre le Créateur.

Le dernier point que je souhaite aborder ici prend appui sur ce que la Bible appelle le sabbat. On sait l'importance de ce septième jour pour la foi juive, où Dieu arrête de travailler et où l'homme est invité à faire de même. Ce qui est signifié ici, entre autres et au-delà du repos nécessaire, c'est que l'homme doit être plus fort que son travail, plus fort que sa force, comme le dit Beauchamp [15]. Ce commandement rejoint bien l'expérience du chercheur: travailler et faire fonctionner ses techniques - aussi sophistiquées soient-elles - ne suffit pas à faire de la bonne recherche. Le véritable scientifique est celui qui sait arrêter son travail pour réfléchir à la fin qu'il poursuit. La connaissance n'est pas le fruit obligé du travail : il y faut le génie, le "temps" de l'intelligence et de l'esprit. Cette nécessité du sabbat est aussi une nécessité éthique. Il nous faut maîtriser le travail scientifique et les techniques qu'il suscite. Il nous faut maîtriser notre maîtrise de la vie et des ressources planétaires. Et cela, on le sait, relève aussi de la responsabilité des scientifiques. La tradition chrétienne a développé cette nécessité de la responsabilité dans le commandement évangélique de la "loi d'amour». C'est bien parce que, selon cette tradition, la responsabilité éthique est bien loin d'être " naturelle » et demeure nécessaire pour que l'humanité reste humaine.

\section{Le débat nécessaire}

Il est possible que telle ou telle position ici tenue soit endossable par d'autres non croyants ou non chrétiens. Il n'y a ici aucune exclusive. S’il y a une originalité et une pertinence à la conception chrétienne, elle sera moins dans le détail de tel ou tel point de vue que dans le sens donné à l'ensemble*. Pourtant mon propos ne consistait pas à résumer l'essentiel de la théologie chrétienne sur la question. Il se veut simplement une contribution à un débat plus que jamais nécessaire. La véritable question n'est pas en effet celle d'une éventuelle réconciliation entre "science » et "foi ", mais bien plutôt celle, pratique et politique, du rôle des scientifiques dans les questions graves de l'heure, en partie posées par les possibilités techniques mises à jour par les chercheurs. La revue médecine/sciences contribue largement à ce débat parmi les biologistes. Y sont requises aussi les ressources authentiques des traditions humaines et culturelles, spirituelles et religieuses, pourvu qu'elles sachent entendre ce qui effectivement se joue dans cette autre tradition humaine de rigueur et d'exigence qu'est la recherche scientifique

\footnotetext{
* On pourra trouver d'autres points de vue complémentaires dans le livre édité par Jean Delumeau [16], dans celui de Thierry Magnin, également prê tre et chercheur scientifique [17], ou bien dans l'ouvrage collectif 118$]$
} 


\section{RÉFÉRENCES}

1. Voir, par exemple, le courrier publié régulièrement dans Nature (pour la période la plus récente : Nature $1993 ; 362$ : $583 ; 363$ : 389-90; $364: 754 ; 366$ : 296 ; 366 : 503)

2. Reeves H. L'Heure de s'eniurer. Paris: Seuil, 1986.

3. Thuan TX. La Mélodie secrète. Paris: Fayard, 1988.

4. Hawking S. Une Brève Histoire du temps. Paris: Flammarion, 1989.

5. Capra F. Le Tao de la physique. Paris: Tchou, 1979.

6. Guitton J, Bogdanov G, Bogdanov I. Dieu et la Science. Paris: Grasset, 1991.

7. Latour B, Woolgar S. La vie de laboratoire. La production des faits scientifiques. Paris: La Découverte, 1988.

8. Bastide F. Le foie lavé. Approche sémiotique d'un texte de sciences expérimentales. Documents du Groupe de recherches sémiolinguistiques, Ecole des Hautes Etudes en Sciences Sociales. Paris, 1979; 1: 7 .

9. Bastide F. La démonstration. Documents du Groupe de recherches sémio-linguistiques, École des Hautes Études en Sciences Sociales $1981 ; 3: 28$

10. Jacob F. La Statue intérieure. Paris: Odile Jacob, 1987: 305-6.

11. Monod J. Le Hasard et la Nécessité. Paris : Seuil, 1970.

12. Beauchamp P. Création et Séparation. Paris: Aubier, Montaigne, Cerf, Delachaux et Niestlé, Desclée de Brouwer, 1969.

13. Gauchet M. Ie Désenchantement du monde. Paris: Gallimard, 1987.

14. Valadier P. L'Église en procès. Paris: Calmann-Lévy, 1987.

15. Beauchamp P. Au commencement, Dieu parle. Etudes 1986; 365: 113-20.

16. Delumeau J. Le Savant et la Foi. Paris: Flammarion, 1989.

17. Magnin T. Quel Dieu pour un monde scientifique? Paris: Nouvelle Cité, 1993.

18. Ouvrage collectif. La Peau de l'âme. Paris : Cerf, 1994.

Philippe Deterre

Directeur de recherche au Cnrs, laboratoire d'immunologie cellulaire et tissulaire (URA Cnrs 625), hôpital Pitié-Salpêtrière, Bat. CERVI, 83, boulevard de l'Hôpital, 75651 Paris Cedex 13, France.

\section{TIRÉS A PART}

P. Deterre 